\title{
RIESGOS A LA SALUD Y AMBIENTE POR EL USO DE LÁMPARAS QUE CONTIENEN MERCURIO*
}

\author{
Ivan Alexander Ayme Huertas \\ Ricardo Cajahuanca Basualdo \\ Rosalyn Daphne Gozar Rafael \\ Sandra Leticia Matos Paredes \\ Neftalí Miranda Miranda \\ Victoria Yolanda Rivera Chale \\ Investigadores independientes \\ Alberto Huiman Cruz \\ Peru Waste Innovation S.A.C
}

\begin{abstract}
Resumen: El presente estudio bibliográfico se desarrolló con el fin de analizar la gestión y manejo de lámparas que contienen mercurio e identificar los riesgos a la salud y ambiente. El mercurio es una sustancia natural y tóxica que una vez liberado al ambiente puede permanecer y circular en el aire, agua y suelo. Asimismo, ocasiona una amplia gama de efectos sistemáticos en los humanos (riñones, estómago, pulmones y sistema nervioso). En el Perú, las lámparas son consideradas como Residuos de Aparatos Eléctricos y Electrónicos (RAEE) al finalizar su vida útil. El problema ambiental que presentan es su contenido en mercurio, no esencial para ningún proceso metabólico y bioacumulable en los seres vivos. Tomando en cuenta el riesgo que representa, se han asumido compromisos por diversos países del mundo, incluyendo Perú, mediante la firma del Convenio de Minamata, los cuales han venido implementando instrumentos de gestión y planificación nacional que permitan llevar a cabo medidas para el correcto manejo de los residuos con mercurio. Como parte de este compromiso asumido, Perú viene elaborando el Plan Nacional de Aplicación del Convenio de Minamata sobre el mercurio, que tiene como propósito concretar acciones a fin de proteger la salud humana y el ambiente de emisiones y liberaciones antropógenas de mercurio y sus compuestos. Se concluye que las lámparas, al finalizar su vida útil, constituyen un peligro debido a su
\end{abstract}

\footnotetext{
* Artículo elaborado en el marco del Curso de Especialización de Residuos del Ámbito Municipal y No Municipal, organizado por el INTE-PUCP y dictado el 2018.
} 
contenido de mercurio, por lo que se requiere un manejo adecuado para reducir los riesgos a la salud humana y ambiente.

Palabras clave: mercurio, lámparas, RAEE, Minamata.

\title{
Health and Environmental Risks Due to the Use of Mercury-Containing Lamps
}

\begin{abstract}
This research was developed in order to analyze the management and management of mercury-containing lamps and to identify the health and environmental risks related to their mercury content. Taking into account that mercury is a natural and toxic substance, which once released into the environment it can remain and circulate in air, water and soil. It also causes a wide range of systematic effects in humans (kidneys, stomach, lungs and nervous system). In Peru, the lamps are considered as waste of electrical and electronic equipment (WEEE) at the end of their useful life. The environmental problem they present is their mercury content, is not essential for any metabolic process and is bioaccumulable in living beings. Taking into account the risk it represents, many countries of the world, including Peru, signed the Minamata Agreement, and have been implementing national planning and management instruments to implement a correct waste management of the mercury. As part of this commitment, Peru has been drafting the National Plan for the Application of the Minamata Convention on Mercury, which aims to specify actions to protect human health and the environment from anthropogenic emissions and releases of mercury and mercury compounds. It concludes that lamps, at the end of their useful life, constitute a hazard due to their mercury it contains; a proper management is an important factor to reduce the risks to human health and the environment.
\end{abstract}

Keywords: Mercury, Lamps, e-waste, Minamata.

\section{Ivan Alexander Ayme Huertas}

Ingeniero Geógrafo por la Universidad Nacional Mayor de San Marcos, con estudio en la Maestría en Gerencia Social en la Universidad Católica del Perú. Cuenta con más de 10 años de experiencias en el sector público como especialista en Gestión de Asuntos Socioambientales, Juventudes y Programas Presupuestales. Actualmente se desempeña como consultor en Estrategias de articulación multisectorial en el Inabif y como facilitador del proceso participativo para la elaboración del PLANMET 2040.

Correo: ivan.ayme@gmail.com 


\section{Ricardo Cajahuanca Basualdo}

Bachiller en Ingeniería Ambiental por la Universidad Peruana Unión (UPeU. Actualmente se desempeña como Promotor Ambiental en la Municipalidad Distrital de Bellavista Callao en el Área de Gestión Ambiental.

Correo electrónico: ricardocb69@gmail.com

\section{Rosalyn Daphne Gozar Rafael}

Ingeniera Ambiental y de Recursos Naturales por la Universidad Nacional del Callao. Actualmente se desempeña como Técnico en gestión de residuos sólidos en el Ministerio del Ambiente.

Correo electrónico: rosalyndg@gmail.com

\section{Sandra Leticia Matos Paredes}

Ingeniera Geógrafa por la Universidad Nacional Mayor de San Marcos. Actualmente se desempeña como Especialista I en Gestión de Residuos Sólidos en la Dirección General de Gestión de Residuos Sólidos del Ministerio del Ambiente.

Correo: sandra.matosp@gmail.com

\section{Neftalí Miranda Miranda}

Bachiller en Ingeniería Ambiental de la Universidad Peruana Unión. Actualmente se desempeña como consultora en gestión de residuos sólidos en el Ministerio del Ambiental.

Correo electrónico: neftaly.miranda1406@gmail.com

\section{Victoria Y. Rivera Chale}

Ingeniera Geógrafa por la Universidad Nacional Mayor de San Marcos, y egresada de la Maestría en Ciencias Ambientales de la misma universidad. Ha trabajado como especialista en sistemas de información geográfica y teledetección en el Instituto del Mar del Perú, como evaluadora de instrumentos de gestión ambiental en el Ministerio de la Producción, y actualmente se desempeña como especialista en gestión de residuos no municipales en el Ministerio del Ambiente. Asimismo, a la fecha representanta al MINAM como miembro del Comité Directivo del Programa Global Industria Sostenible de Reciclaje (SRI) - 2da Fase a cargo de la Cooperación Suiza, programa relacionado con el adecuado manejo de los residuos de aparatos eléctricos y electrónicos.

Correo electrónico: vickyriverachale@gmail.com

\section{Alberto Huiman Cruz}

Ingeniero Geógrafo, Magíster en Ciencias Ambientales con Mención en Gestión y Ordenamiento Ambiental del Territorio, y Doctorando en Ciencias Ambientales. Calificado como Experto Internacional de Residuos, por la International Solid Waste Association (ISWA). Expositor, representando al Perú, en tema de residuos sólidos en Costa Rica, El Salvador, Colombia y Argentina. Actual Gerente General de Peru Waste Innovation S.A.C.

Correo electrónico: alberto@pwi.com.pe 


\section{Introducción}

La presente revisión bibliográfica se desarrolló con el objetivo de analizar el manejo de los residuos de lámparas con contenido de mercurio y conocer los riesgos a la salud humana y al ambiente relacionados al manejo de este residuo. Así como conocer las alternativas de manejo de las lámparas fluorescentes con mercurio de los países citados y encontrar la alternativa de manejo desde el punto de vista ambiental y económicamente más factible de implementación en el país, con el propósito de minimizar el volumen del residuo y facilitar su disposición final.

Las lámparas fluorescentes de tubo, de uso común y utilizadas frecuentemente para iluminación tanto en domicilios, industrias, comercios e instituciones, una vez empleadas son consideradas como residuo peligroso debido al contenido de mercurio presente; al tener un manejo inadecuado, pueden ocasionar impactos a la salud humana y al ambiente (EPA 1994).

Actualmente en el país, las lámparas fluorescentes son consideradas como residuos de aparatos eléctricos y electrónicos y dispuestas como residuo convencional, debido al desconocimiento del problema que presentan al final de su vida útil y los altos costos que implica el tratamiento y la disposición final (Vargas 2017).

Poulin y Gibb (2008) señalan que el mercurio (Hg) es un elemento metálico que está presente en la naturaleza. Por ser uno de los elementos conocidos desde tiempos inmemoriales, el mercurio se ha usado históricamente con distintos fines. Sin embargo, las liberaciones de mercurio han ido en aumento desde la revolución industrial, debido fundamentalmente a la extracción de oro artesanal y en pequeña escala, la generación de energía y los procesos industriales, lo que ha causado un aumento de la contaminación ambiental mundial con mercurio (Gaona 2004).

Hoy en día sabemos que el mercurio es causa importante de efectos neurológicos y de otra índole en la salud de los seres humanos y en otras formas de vida. Tan pronto se libera al ambiente, el mercurio persiste y circula en la atmósfera, el agua, el suelo y la biota. El mercurio puede ser absorbido por los microorganismos y se va acumulando hasta los niveles superiores de la cadena trófica (PNUMA 2002). 
Además, el mercurio puede ser transportado en la atmósfera a largas distancias, circunstancia que justificó la elaboración de un convenio mundial (Convenio de Minamata) para reducir los peligros que entraña la contaminación con mercurio (OMS 2017).

Algunos países, como Argentina, Brasil, El Salvador, España, Estados Unidos, entre otros, vienen implementando estudios sobre el tema, donde la experiencia muestra que la segregación en la fuente de este tipo de residuo marca la eficacia de los planes de gestión. Los Estados Unidos marca la pauta en cuanto al manejo de lámparas fluorescentes de tubo desechadas desde el punto de vista de residuos peligrosos, por cuanto ha definido políticas e instrumentos para la gestión de estas luminarias (PNUMA 2017).

\section{Metodología}

Este estudio recoge información de fuentes bibliográficas relacionadas al tema, así como la normativa peruana sobre los residuos de aparatos eléctricos y electrónicos y su manejo, en especial lo referido al mercurio.

Con la información seleccionada, se procedió a identificar casos emblemáticos sobre la inadecuada gestión del mercurio, luego de una breve discusión grupal fueron seleccionados dos: caso Minamata (Japón) y caso Choropampa (Perú). La selección de ambos se debió a que fueron punto de partida para el fortalecimiento de políticas a nivel internacional y nacional, respectivamente, para eliminar o minimizar los riesgos a la salud humana y al ambiente derivados del mercurio.

Luego se evaluó la información de los artículos e investigaciones científicas sobre el manejo y gestión de lámparas fluorescentes con contenido de mercurio, los aspectos de riesgo del mercurio y, tras debate en grupo, se elaboró un cuadro de experiencias en el manejo de mercurio y su impacto en la salud humana y el ambiente. Finalmente, concluimos y formulamos propuestas como recomendaciones a la mejora en relación al tema de investigación.

\section{El Convenio de Minamata}

Como respuesta a la preocupación internacional sobre el mercurio, se publicó en el año 2002 la primera evaluación mundial sobre el mercurio y sus compuestos. Ante la evidencia del impacto global de esta sustancia, se creó en 2005 la Asociación Mundial sobre el Mercurio del PNUMA.

Como resultado de las diferentes evaluaciones, y ante la necesidad urgente de adoptar medidas a nivel internacional, en febrero de 2009 el 
entonces Consejo de Administración del PNUMA decidió iniciar el proceso de negociación de un instrumento jurídicamente vinculante sobre el mercurio.

Dicho proceso tuvo lugar durante un periodo de tres años (20102013), hasta que el Comité Intergubernamental de Negociación (CIN), creado a tal efecto, acordó en enero de 2013 el texto del «Convenio de Minamata sobre Mercurio», en alusión al episodio de contaminación que tuvo lugar en esta localidad de Japón. El texto fue adoptado formalmente durante la Conferencia de Plenipotenciarios, que tuvo lugar en Japón en octubre de 2013. A la fecha, 128 países han suscrito dicho convenio y 107 países han ratificado el mismo.

El «Convenio de Minamata sobre Mercurio» contiene las metas y mecanismos internacionales para facilitar las acciones que los países realicen para prevenir emisiones y vertimientos de mercurio que ponen en riesgo la salud humana y el medio ambiente en todo el mundo.

El objetivo del Convenio de Minamata es proteger la salud humana y el medio ambiente de las emisiones y liberaciones antropógenas de mercurio y sus componentes, para ello contiene disposiciones sobre:

- $\quad$ El suministro y comercio internacional de mercurio.

- $\quad$ Reducción progresiva del uso de mercurio en productos y procesos.

- Mecanismos financieros para la implementación del Convenio y el desarrollo de capacidades, asistencia técnica y transferencia tecnológica.

- La protección y vigilancia de la salud, enfocadas en las poblaciones en situación de riesgo, particularmente en las poblaciones vulnerables.

- Orientaciones para la implementación de planes de acciones nacionales sobre reducción de uso del mercurio en minería artesanal y pequeña minería.

El Convenio prevé la reducción gradual de productos y procesos, o componentes de un producto al que se haya añadido mercurio o un compuesto de mercurio de manera intencional.

Estos productos con mercurio añadido se agrupan en acumuladores y baterías; relés y antisépticos de uso tópico; aparatos e instrumental de medición (termómetros, barómetros, higrómetros, manómetros y esfigmomanómetros); y cosméticos y jabones para aclarar la piel.

\section{- Minamata y el compromiso nacional}

El Convenio de Minamata fue firmado por Perú el 10 de octubre de 2013, aprobado con resolución legislativa 30352 y ratificado con decreto supremo 
061-2015-RE el 25 de noviembre de 2015. Asimismo, mediante decreto supremo 010-2016-MINAM se aprobó el Plan de Acción Multisectorial para la implementación del Convenio de Minamata sobre Mercurio.

Durante 2019, el Ministerio del Ambiente de Perú (MINAM) elaboró el Plan Nacional de Aplicación del Convenio de Minamata sobre Mercurio, el cual definirá una serie de resultados, así como indicadores de cumplimiento, necesarios para la medición, reporte y verificación.

\section{Gestión y manejo de los residuos de aparatos eléctricos y electrónicos (RAEE) en el Perú}

En el año 2012, mediante decreto supremo 001-2012-MINAM, se aprobó el Reglamento Nacional para la Gestión y Manejo de los RAEE, a través del cual se establecieron un conjunto de derechos y obligaciones para los actores involucrados (productores, operadores, municipalidades y consumidores), a fin de realizar una adecuada gestión y manejo ambiental de los RAEE. Cabe señalar que el reglamento considera diez categorías de Aparatos Eléctricos y Electrónicos (AEE), de las cuales, la categoría cinco comprende a los aparatos de alumbrado, incluidas las lámparas.

Asimismo, se definió el rol de dichos actores, en el cual resalta la figura del productor, con la finalidad de que asuma su responsabilidad en distintas etapas de este manejo, como parte de un sistema de responsabilidad extendida y compartida, y en el marco de la logística inversa.

De acuerdo al mencionado reglamento, el Ministerio del Ambiente quedaba facultado para dictar las disposiciones complementarias que fueran necesarias para su mejor aplicación. Es así que, mediante resolución ministerial 200-2015-MINAM, se aprobaron un conjunto de disposiciones complementarias que regulan la meta anual de manejo de RAEE contenida en los planes de manejo para las categorías tres y cuatro (para la categoría cinco se mantiene voluntaria), la presentación de los planes de manejo de RAEE para todas las categorías, y la temporalidad para la presentación de la declaración anual de productores de AEE y operadores de RAEE.

Al 2019, Perú cuenta con Normas Técnicas Peruanas respecto a la adecuada gestión de los RAEE, entre ellas, se tienen las siguientes: generalidades, tratamiento o manejo, y centros de acopio.

Con la aprobación del reglamento, se sentaron las bases de la Responsabilidad Extendida del Productor (REP), principio que forma parte del Régimen Especial de Bienes Priorizados establecido en el decreto legislativo 1278, Ley de Gestión Integral de Residuos Sólidos (LGIRS), orientado a aquellos bienes de consumo masivo que directa o indirectamente 


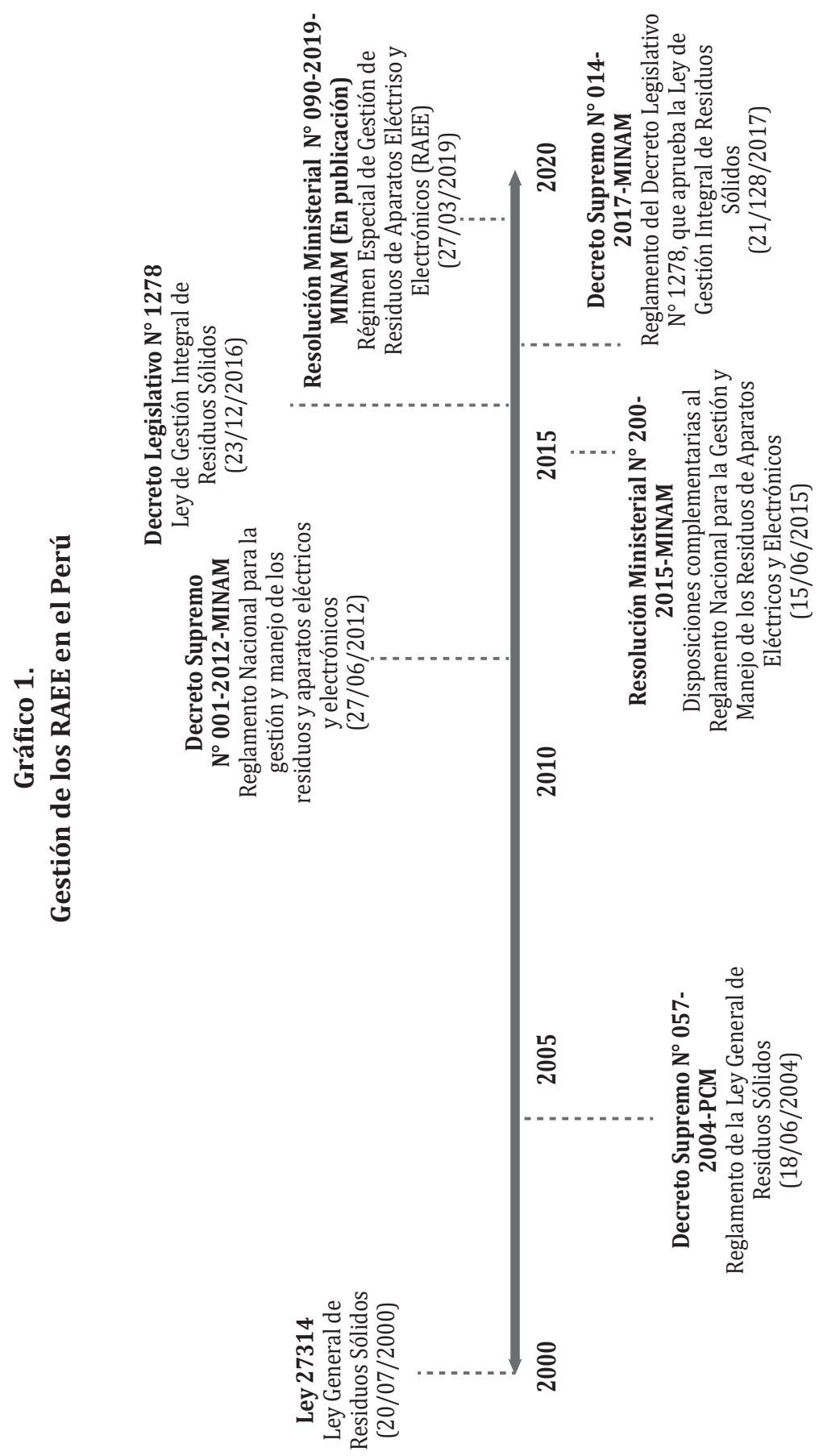


inciden significativamente en la generación de residuos sólidos en volúmenes considerables o que por sus características de peligrosidad requieran de un manejo especial.

En virtud de este régimen especial, los productores deben asumir responsabilidad en las etapas de post consumo de estos bienes, la cual se traduce en la implementación de sistemas individuales o colectivos destinados a la recuperación y valorización de estos residuos.

En el año 2018, a fin de adecuar el Reglamento Nacional para la Gestión y Manejo de los RAEE al Régimen Especial de Bienes Priorizados establecido en la LGIRS, el Ministerio del Ambiente elaboró el proyecto normativo Régimen Especial de Gestión de RAEE, el cual se encuentra en proceso de consulta pública para su posterior aprobación.

Para identificar un bien priorizado, este debe cumplir con ciertos criterios establecidos en el Reglamento de la referida Ley, aprobado mediante decreto supremo 014-2017-MINAM, de los cuales, los RAEE cumplen con tres de ellos: el volumen de generación de los residuos, las posibilidades de valorización de los mismos y la peligrosidad de sus componentes.

Respecto al manejo de los residuos de lámparas en el Perú (categoría cinco) al 2019, no existe un manejo diferenciado de estos, pues a nivel nacional las plantas de valorización de RAEE aún no cuentan con tecnología adecuada para realizar un tratamiento específico y aprovechamiento de los materiales; por lo que dichos RAEE son gestionados a través del servicio municipal y, en el mejor de los casos, son dispuestos en un relleno de seguridad como residuos peligrosos.

Gráfico 2.

\section{Manejo de los RAEE en el Perú}

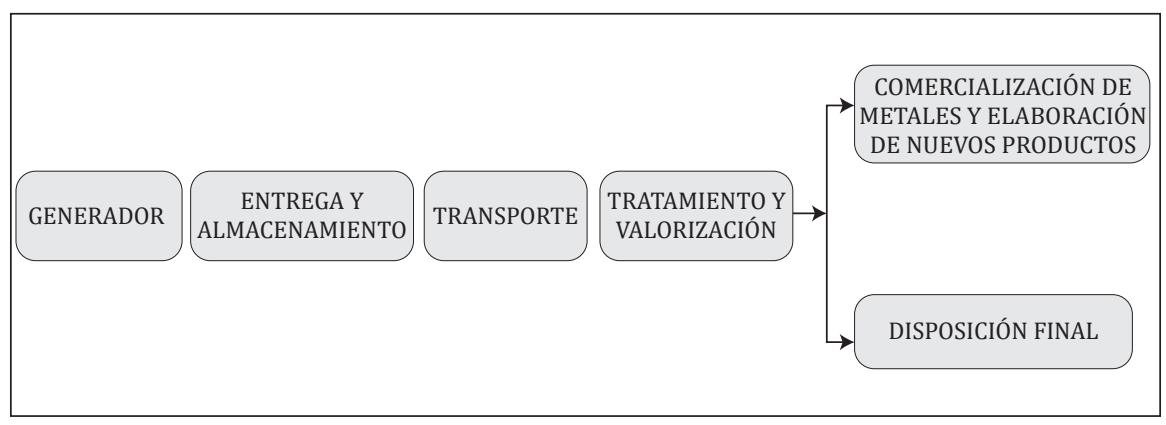

Elaboración propia. 
Tabla 1.

Empresas operadoras que manejan RAEE en el Perú

\begin{tabular}{|l|c|c|c|}
\hline \multirow{2}{*}{} & \multicolumn{2}{|c|}{ Registro DIGESA } & Registro MINAM \\
\cline { 2 - 4 } & EPS-RS & EC-RS & EO-RS \\
\hline Lima & 29 & 33 & 46 \\
\hline Callao & 4 & 1 & 11 \\
\hline Ancash & 1 & - & 3 \\
\hline Arequipa & 1 & - & 2 \\
\hline Ayacucho & - & - & 4 \\
\hline Cajamarca & - & 2 & 2 \\
\hline Cusco & 2 & 2 & 2 \\
\hline Huancavelica & - & - & 2 \\
\hline Ica & - & - & 3 \\
\hline Junín & - & - & 1 \\
\hline La Libertad & 1 & - & 6 \\
\hline Lambayeque & - & 1 & 1 \\
\hline Madre de Dios & - & - & 4 \\
\hline Piura & - & - & - \\
\hline San Martín & - & - & - \\
\hline Tacna & - & $\mathbf{4 1}$ & 2 \\
\hline Tumbes & $\mathbf{3 9}$ & - & 2 \\
\hline Ucayali & - & - & 2 \\
\hline Total & - & - & 2 \\
\hline
\end{tabular}

Fuente: Elaboración propia, adaptado del Registro de EC-RS (DIGESA 2019), Registro de EPSRS (DIGESA 2019) y Listado de empresas operadoras de residuos sólidos autorizados por el MINAM (MINAM 2019).

\section{- Empresas operadoras de residuos de aparatos eléctricos y electrónicos en el Perú}

Las empresas operadoras de residuos sólidos (EO-RS) que brindan servicios relacionados al manejo de residuos de aparatos eléctricos y electrónicos (RAEE), las constituyen el total de empresas comercializadoras de residuos sólidos (EC-RS) ${ }^{1}$ y empresas prestadoras de servicios de residuos sólidos 
(EPS-RS) ${ }^{2}$ con registro vigente ante la Dirección General de Salud Ambiental e Inocuidad Alimentaria (DIGESA) y las EO-RS registradas ante el Ministerio del Ambiente (MINAM) con operaciones autorizadas relacionadas al manejo de RAEE.

Las empresas operadoras registradas en DIGESA, hasta diciembre de 2017, son 39 EPS-RS RAEE y 41 EC-RS RAEE. De acuerdo al Listado de empresas operadoras de residuos sólidos autorizadas por el MINAM, hasta marzo de 2019 se cuenta con 92 empresas operadoras de residuos sólidos con fines de prestación de servicios y comercialización con autorización para operaciones de recolección y transporte de RAEE (MINAM 2019).

\section{Lámparas}

Las lámparas de descarga son fuentes luminosas que generan luz mediante una descarga eléctrica, la misma que se produce por dos electrodos al interior de un tubo lleno de gas; por otro lado, las lámparas incandescentes emiten luz debido a las altas temperaturas que alcanza el filamento en su interior. Las lámparas de descarga se clasifican de acuerdo al gas que usan y a la presión al interior del tubo de descarga (ELT citado por Miranda, Martínez \& Hernández 2015).

\section{Figura 1.}

Tipos de lámparas que contienen mercurio

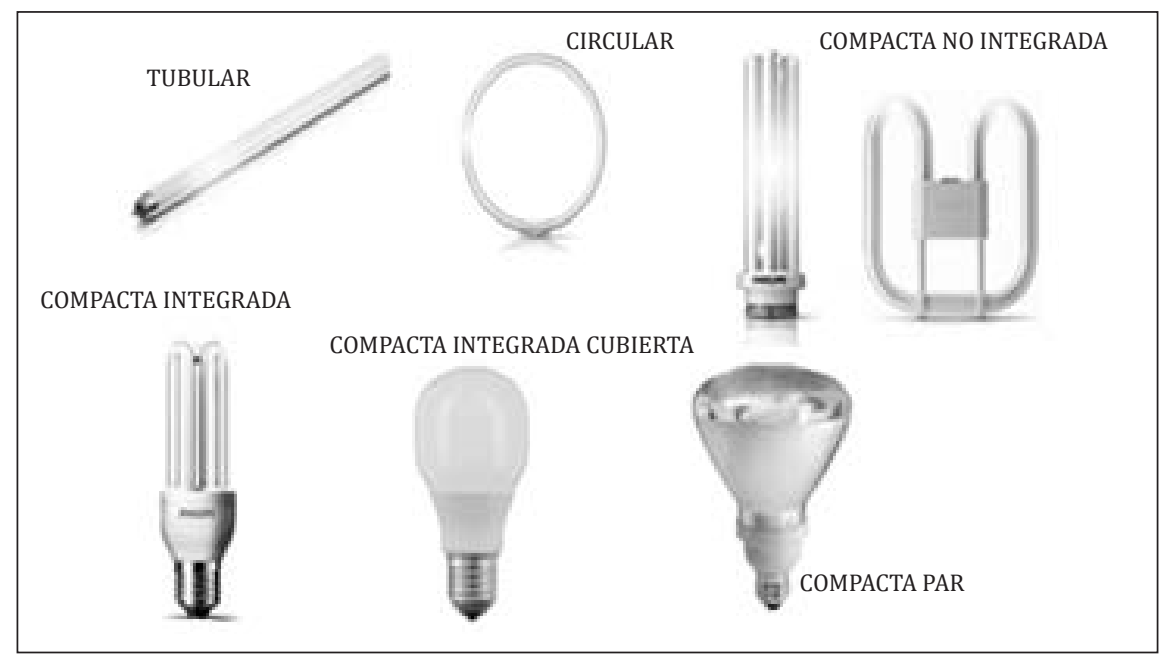

Fuente: European Lamp Companies Federation citado en Miranda et al. (2015).

2 Este sistema de clasificación ya no existe. 
Las lámparas de descarga de baja presión, excepto la de sodio de baja presión, y las lámparas de descarga de alta presión, contienen en su interior vapor de mercurio. El contenido de mercurio en las lámparas de descarga de baja y alta presión dependen del tipo de lámpara y fabricante (Martineza, Ayrala \& Zurbriggen 2012). El contenido referido se muestra en la siguiente tabla.

Tabla 2.

Tipos de lámparas y su contenido de mercurio

\begin{tabular}{|l|c|}
\hline \multicolumn{1}{|c|}{ Tipo de lámpara } & Contenido de mercurio en mg \\
\hline Incandescente & No contiene \\
\hline Incandescente halógena & No contiene \\
\hline Fluorescente tubular & 15 \\
\hline Fluorescente compacta & 5 \\
\hline Mercurio Alta Presión & 30 \\
\hline MAP con halogenuros metálicos & 30 \\
\hline Sodio Alta Presión & 25 \\
\hline Sodio Baja Presión & No contiene \\
\hline
\end{tabular}

Fuente: European Lamp Companies Federation citado en Miranda et al. (2015).

En los Estados Unidos las lámparas fluorescentes han sido declaradas por la Agencia de Protección Ambiental (Environmental Protection Agency, EPA) como residuos peligrosos, los mismos que reciben un manejo más riguroso, además de contener otros 17 elementos peligrosos, esto según la Ley de conservación y Recuperación de Residuos. Asimismo, la Universidad de Granada (UGR) (citada por Miranda et al. 2015) sostiene que el mercurio es el elemento más tóxico de todos los metales, no es esencial para ningún proceso metabólico y es bioacumulable en la mayoría de los seres vivos.

De acuerdo al estudio realizado en el Perú por el Instituto Cuanto (2015), la cantidad promedio de compra de «focos» anualmente es de 3,1 focos. El lugar de preferencia para la compra de las lámparas a nivel nacional son las ferreterías $(60,7 \%)$, seguido de bodegas y supermercados. Asimismo, el motivo de la compra es por reemplazo (90\%) y la demanda anual es de 26.473.279 unidades.

\section{Mercurio}

Según Gaona (2004), el mercurio (Hg) es un metal pesado de coloración blanco-plateado y líquido a temperatura y presión ambiente. Está presente 
de manera natural en la corteza terrestre, y puede ser transportado en el ambiente por el aire y el agua (Poulin \& Gibb 2008).

Se libera a la atmósfera en forma de vapor por fenómenos naturales, como la actividad volcánica, los incendios forestales, el movimiento de masas de agua, la erosión de rocas y procesos biológicos. Asimismo, las fuentes antropogénicas de mercurio contribuyen significativamente a las concentraciones ambientales de este y comprenden las operaciones de minería, los procesos industriales, la combustión de combustibles fósiles, la producción de cemento y la incineración de residuos sanitarios, químicos y municipales (Poulin \& Gibb 2008).

El mercurio posee tres formas: elemental (o metálico), inorgánico (al que la persona se puede ver expuesta en ciertos trabajos) y orgánico (como el metilmercurio, que penetra en el cuerpo humano por vía alimentaria). Estas formas de mercurio difieren por su grado de toxicidad y sus efectos sobre los sistemas nervioso e inmunitario, el aparato digestivo, la piel, los pulmones y los ojos (OMS 2017).

Según el Ministerio de Vivienda, Ordenamiento Territorial y Medio Ambiente de Uruguay (MVOTMA 2017), el mercurio, debido a sus excelentes propiedades físicas como dilatación térmica y conductividad eléctrica, se usa en pilas, aparatos de medición como termómetros, barómetros y manómetros, interruptores de mercurio, lámparas fluorescentes compactas, lámparas de alumbrado público y otros dispositivos eléctricos.

Tabla 3.

Contenido de mercurio en distintos dispositivos

\begin{tabular}{|l|l|}
\hline \multicolumn{1}{|c|}{ Tipo de dispositivo } & \multicolumn{1}{c|}{$\begin{array}{c}\text { Rango de contenido de } \\
\text { mercurio }\end{array}$} \\
\hline $\begin{array}{l}\text { Lámparas fluorescentes compactas de uso } \\
\text { doméstico }\end{array}$ & De 1 a $5 \mathrm{mg}$ \\
\hline $\begin{array}{l}\text { Lámparas de alumbrado público - de vapor de } \\
\text { sodio y/o mercurio }\end{array}$ & $\begin{array}{l}\text { De } 75 \text { vatios - hasta } 25 \mathrm{mg} \\
\text { De } 1500 \text { vatios - hasta } 225 \mathrm{mg}\end{array}$ \\
\hline Termómetros clínicos & De 50 a $1500 \mathrm{mg}$ \\
\hline Termómetros industriales y de laboratorio & De 500 a $4000 \mathrm{mg}$ \\
\hline Barómetros, manómetros y vacuómetros & De 30000 a $60000 \mathrm{mg}$ \\
\hline Medidores de flujo, indicadores de consumo & Hasta $50000 \mathrm{mg}$ \\
\hline
\end{tabular}

Elaboración propia, adaptado de MVOTMA (2017). 
REVISTA KAWSAYPACHA: SOCIEDAD Y MEDIO AMBIENTE Nº 4 (2019)

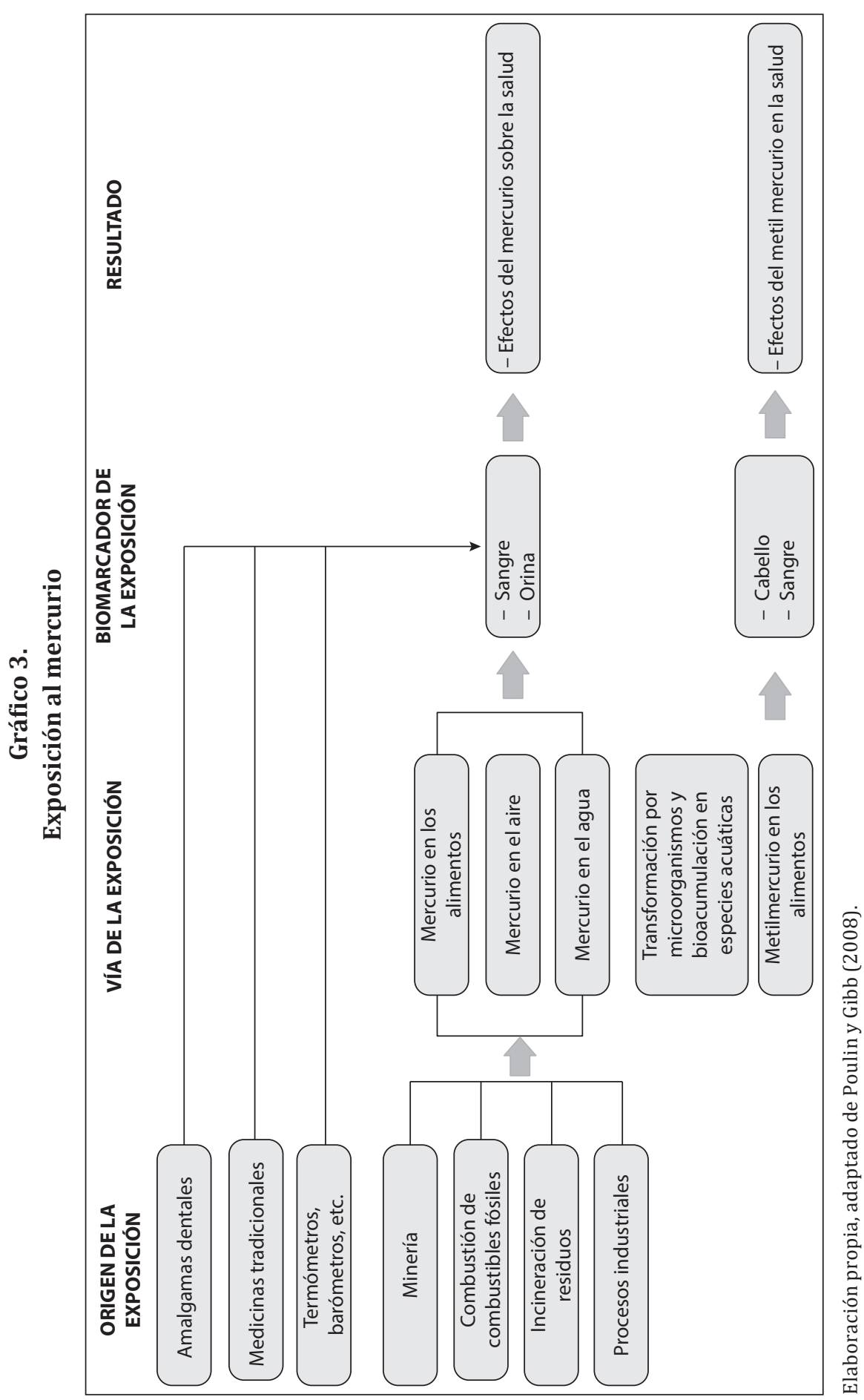




\subsection{Mercurio inorgánico}

Poulin y Gibb (2008) mencionan que la exposición de la población a especies inorgánicas de mercurio puede producirse a partir de diversas fuentes. Estas son algunas de las principales:

- El contacto con productos que contienen mercurio (como termómetros, barómetros, termostatos, interruptores eléctricos, lámparas fluorescentes, entre otros).

- La exposición a amalgamas de empastes dentales.

- $\quad$ La inhalación de aire cerca de refinerías y minas de mercurio, plantas industriales en las que se utilice este o lugares en los que se hayan aplicado fungicidas que lo contengan.

- $\quad$ En el caso de los niños, los juegos en terrenos contaminados o con el mercurio de termómetros.

- $\quad$ El uso de medicamentos tradicionales o herbarios (como antisépticos, diuréticos o laxantes).

\section{Efectos a la salud y al ambiente por mercurio}

\subsection{Fuentes de exposición}

Gaioli, Amoedo y Gonzales (2012) indican que la atmósfera, el agua y el suelo son los receptores de las liberaciones de mercurio en el ambiente. Las principales fuentes de liberación de mercurio son las fuentes naturales, debido a la movilización natural del mercurio en la corteza terrestre por actividad volcánica o erosión de rocas; o de liberaciones antropogénicas resultantes de la presencia de mercurio en materias primas como los combustibles fósiles (carbón, gas, petróleo y otros minerales extraídos, tratados y reciclados), siendo el $85 \%$ de emisiones antropogénicas las que provienen de esta fuente. También producen liberaciones las industrias cloroalcalinas de fabricación de papel, instrumental médico (termómetros, amalgamas dentales), termostatos, lámparas fluorescentes, cementeras, faros de automóviles, tratamiento de desechos, vertederos, cremación y pinturas.

En el agua y en el suelo, bacterias metanogénicas transforman el mercurio en metilmercurio [CH3Hg]+, la forma más tóxica de este metal, siendo este compuesto de fácil acumulación en la cadena trófica animal. El metilmercurio se une a las proteínas de las algas y del plancton, siendo los pescados y mariscos la principal fuente de exposición para el hombre (Gaioli et al. citados por Ortega et al. 2003). 


\subsection{Toxicología del mercurio}

Según SCHER (2010), las personas, luego de la rotura de una lámpara fluorescente en un área cerrada, pueden estar expuestas al mercurio por inhalación e ingestión oral; después de ser inhalado, más del $80 \%$ de vapor de mercurio es absorbido por los pulmones, sin embargo, el mercurio ingerido es mal absorbido por el tracto intestinal (menos del 0,01\%). La eliminación del mercurio después de la inhalación es lenta (vida media de 60 días) y la mayoría se elimina a través de la orina (como iones de mercurio) y heces, siendo una pequeña cantidad de mercurio eliminado a través de la exhalación y el sudor.

Aucott, McLinden y Winka (2003) desarrollaron en los Estados Unidos un experimento en un escenario en el cual se desecharon lámparas fluorescentes compactas de bajo contenido de mercurio $(4,55 \mathrm{mg})$ rotas, las cuales fueron almacenadas en un contenedor descubierto por un periodo de tiempo antes de su disposición final, con el objetivo que conocer la cantidad de mercurio que se libera de las lámparas a tres temperaturas diferentes, utilizando un equipo de detector de vapor de mercurio. Tomando como indicadores la cantidad de mercurio liberado por periodo de tiempo y la velocidad de liberación en gramos por hora. De acuerdo a esta investigación, la tasa de liberación de mercurio varía proporcionalmente con la temperatura, debido a la mayor volatilidad del mercurio a temperaturas más altas, concluyendo que a temperaturas que van de $40^{\circ} \mathrm{F}$ a $85^{\circ} \mathrm{F}\left(4,5^{\circ} \mathrm{C}\right.$ a $\left.29^{\circ} \mathrm{C}\right)$ se volatiliza aproximadamente $17 \%$ a $40 \%$ del mercurio contenido, en un periodo de dos semanas. A partir del estudio realizado, Aucott et al. (2003) indican que las lámparas desechadas liberan aproximadamente de dos a cuatro toneladas/año de mercurio en el caso de los Estados Unidos, tomando en cuenta que en este país se desechan aproximadamente 620 millones de lámparas fluorescentes compactas anualmente y solo el $20 \%$ de ellas se recicla a nivel nacional. En cuanto a la emisión de mercurio al ambiente, el estudio sugiere que los niveles elevados de mercurio en el aire superan la concentración de referencia de la EPA, de $300 \mathrm{ng} / \mathrm{m}^{3}$, en las proximidades a donde se almacenaron las lámparas rotas, existiendo un potencial riesgo sobre trabajadores o personas que participan del reciclaje de estas y que estén en contacto con lámparas rotas.

\subsection{Efectos sobre la salud}

Gaioli et al. (2012) indican que el vapor de mercurio se absorbe rápidamente en los pulmones (75-85\% de la dosis inhalada) y en forma líquida apenas se absorbe por la vía gastrointestinal $(0,01 \%)$. El mercurio se oxida a ion 
mercurio, perdiendo capacidad de difundirse, quedando retenido en glóbulos rojos, sistema nervioso central y riñones.

Los niños y el feto en etapas de desarrollo son más vulnerables que los adultos, debido que el mercurio inhibe la división celular y la migración durante el desarrollo (Rice \& Barone citado por SCHER 2010).

La vía de excreción más importante es la digestiva y en menor porcentaje la urinaria, respiratoria y sudorípara (Gaioli et al. 2012).

Según Gaioli et al. (2012), la principal vía de exposición es la oral, aunque también puede producirse a través de la vía respiratoria; el mercurio puede ser medido o detectado en varias muestras (sangre, orina, pelo).

\section{Daños ocurridos por mercurio}

\subsection{A nivel internacional: Caso Minamata}

La ciudad de Minamata (Japón) es un pequeño pueblo pesquero ubicado en la bahía de Minamata, donde a partir de los años treinta se instaló e inició operaciones la industria Chisso Corporation. Como parte de sus operaciones, la empresa empezó a realizar vertimientos con compuestos de mercurio. Inicialmente se fueron detectando anomalías en comportamiento y muerte de gatos, perros, aves y peces, pero no fue sino hasta 1956 que se detectaron los primeros casos en personas: 46 murieron. La cifra aumentó hasta el año 1965, cuando se contabilizaron 111 víctimas y más de 400 casos con problemas neurológicos, sin que inicialmente se supiera la causa. Asimismo, madres que no presentaban ningún síntoma dieron a luz niños gravemente afectados.

Por ello, luego de investigaciones, en 1968 el Gobierno japonés anunció oficialmente que la causa de la enfermedad era la ingesta de pescados y mariscos contaminados con mercurio, provocado por los vertidos de la empresa petroquímica Chisso.

Se calcula que entre 1932 y 1968, año en que cambió el proceso industrial en Chisso por otro menos contaminante, se vertieron a la bahía 81 toneladas de mercurio. Según información del Ministerio de Medio Ambiente de Japón, hasta finales de mayo de 2013, el número total de pacientes certificados era de 2.977, de los cuales 646 seguían con vida.

La empresa responsable de los vertidos continúa pagando grandes sumas de dinero en indemnizaciones, mientras que el Gobierno japonés sigue implementando medidas para mitigar las secuelas de esta contaminación, como el subsidio permanente de los gastos médicos de las víctimas, la indemnización basada en un acuerdo para víctimas certificadas según normativa específica, entre otras. 


\subsection{A nivel de Perú: Caso Choropampa}

En Cajamarca, situada en los Andes del norte de Perú, se ubica la Minera Yanacocha, la mina de oro más grande de América Latina. El día viernes 2 de junio del año 2000, un camión de transporte produjo el derrame de 151 kg de mercurio metálico; más de un millar de campesinos y campesinas que no conocían los efectos tóxicos del mercurio fueron afectados por este accidente.

La absorción del vapor de mercurio en el medio ambiente y exposición directa produjo a los pobladores diversos síntomas tales como sabor metálico en la boca, problemas respiratorios, sarpullido, labilidad emocional, insomnio, pérdida de la memoria, cambios en el sistema neuromuscular, dolores de cabeza, dolor lumbar, entre otros. Asimismo, 935 personas resultaron con niveles de mercurio en la sangre por encima del máximo recomendado.

Después de 18 años del suceso, la existencia de centenares de testimonios de pobladores que fueron expuestos al mercurio y los recientes informes de vigilancia de la DIGESA, muestra que la situación de salud sigue siendo un problema que no ha sido superado porque aún los comuneros siguen sufriendo las consecuencias en su salud.

Hasta el año 2004 no existía en Perú una ley que regule el transporte de sustancias tóxicas, por lo que el traslado de sustancias como el mercurio estaba únicamente sujeto a la autorregulación de las empresas, tal es el caso de la Minera Yanacocha SRL y su transportista RANSA, quienes no adoptaron ninguna medida de seguridad.

\section{Experiencias en el manejo y gestión de mercurio de lámparas}

\subsection{América Latina y El Caribe}

\begin{tabular}{|c|l|}
\hline \multirow{5}{*}{ Perú } & Se cuenta con legislación que regula el manejo post consumo de lámparas, \\
las mismas que se encuentran catalogadas dentro de la categoría 5.- Aparatos \\
de alumbrado, del Reglamento Nacional para la Gestión y Manejo de los \\
Residuos, Aparatos Eléctricos y Electrónicos. Asimismo, dentro de la Ley \\
de Gestión Integral de Residuos Sólidos y su Reglamento, se regula a los \\
«Bienes priorizados» donde los RAEE estarían siendo considerados. Además, \\
actualmente, en el Perú los productores de aparatos de alumbrado no cuentan \\
con un Plan de Manejo de RAEE para lámparas, quienes, bajo el principio de \\
Responsabilidad Extendida del Productor (REP), se encargan del manejo y \\
gestión de este tipo de residuos.
\end{tabular}




\begin{tabular}{|c|c|}
\hline Argentina & $\begin{array}{l}\text { El marco que regula el manejo de los residuos en Argentina se fundamenta en } \\
\text { la Ley General del Ambiente. La legislación vigente no incluye disposiciones } \\
\text { específicas respecto a la gestión de los residuos de mercurio, por lo tanto, } \\
\text { son regulados mediante la Ley de Residuos Peligrosos. En ese sentido, las } \\
\text { lámparas fluorescentes se recogen como residuo peligroso, aunque no } \\
\text { existe un sistema uniforme de recogida de dichos residuos. Dentro de las } \\
\text { instalaciones de tratamiento de residuos peligrosos, una trituradora reduce } \\
\text { el volumen de los desechos de las lámparas; mientras que los materiales } \\
\text { reciclables, tales como el aluminio y el vidrio, se venden en los mercados } \\
\text { secundarios. Los residuos que contienen mercurio se envían a vertederos } \\
\text { especialmente diseñados. }\end{array}$ \\
\hline Bolivia & $\begin{array}{l}\text { La política nacional determina que los residuos que contienen mercurio, } \\
\text { como las lámparas fluorescentes, deben tratarse según la logística inversa, } \\
\text { una herramienta que sirve para aplicar el principio de responsabilidad } \\
\text { compartida a lo largo del ciclo de vida del producto. Actualmente, Bolivia } \\
\text { no cuenta con un sistema nacional de recolección de lámparas fluorescentes } \\
\text { y focos ahorradores, ni con instalaciones de tratamiento y eliminación, } \\
\text { por lo que la gestión de los residuos de mercurio todavía es un desafío. Es } \\
\text { importante señalar que se tiene un prototipo de triturador portátil que } \\
\text { permite triturar, separar y aislar el mercurio presente en las lámparas } \\
\text { fluorescentes, reduciendo así los efectos perjudiciales de la contaminación } \\
\text { que puede originar el mercurio al romperse el exterior de estas lámparas. }\end{array}$ \\
\hline Brasil & $\begin{array}{l}\text { Los desechos de mercurio se clasifican como peligrosos, y se aplican } \\
\text { procedimientos y obligaciones especiales en relación con su almacenamiento, } \\
\text { transporte, tratamiento y eliminación. La Política Nacional de Gestión de } \\
\text { Residuos especifica que determinados residuos que contienen mercurio, } \\
\text { como las lámparas fluorescentes, deben tratarse según la logística inversa. } \\
\text { El país dispone de la capacidad técnica necesaria para tratar correctamente } \\
\text { las lámparas que contienen mercurio. Empresas especializadas se ocupan } \\
\text { de la recogida y reciclaje de los materiales no peligrosos, la recuperación del } \\
\text { mercurio y la eliminación. Los residuos peligrosos, entre ellos los residuos de } \\
\text { mercurio, se envían a vertederos especiales. }\end{array}$ \\
\hline Chile & $\begin{array}{l}\text { Cuenta con la Estrategia de Iluminación Eficiente para Chile, en la cual se } \\
\text { señala como principales lineamientos: } \\
\text { a) Promover la innovación tecnológica, habilitando la adopción de } \\
\text { productos eficientes de iluminación. } \\
\text { b) Avanzar en el cumplimiento de la reducción de } 12 \% \text { de la demanda de } \\
\text { energía al } 2020 \text { según el Plan de Acción de Eficiencia Energética } 2020 \\
\text { (PAEE20). } \\
\text { c) Aportar a la reducción de emisiones de gases de efecto invernadero. } \\
\text { d) Controlar los niveles de mercurio en los productos de iluminación y } \\
\text { asegurar su disposición adecuada al final de la vida útil, actuando acorde } \\
\text { con los compromisos que el país está evaluando adquirir ante la firma } \\
\text { del Convenio de Minamata. }\end{array}$ \\
\hline México & $\begin{array}{l}\text { En México existen } 63 \text { empresas autorizadas por la SEMARNAT para la } \\
\text { recolección y transporte de lámparas fluorescentes y } 71 \text { empresas autorizadas } \\
\text { para el almacenamiento (acopio) de estas lámparas. Adicionalmente, la } \\
\text { empresa SITRASA, S.A. de C.V., ubicada en la ciudad de Irapuato, Guanajuato, } \\
\text { tiene autorizado el tratamiento de residuos peligrosos de lámparas } \\
\text { (SEMARNAT 2014). }\end{array}$ \\
\hline
\end{tabular}




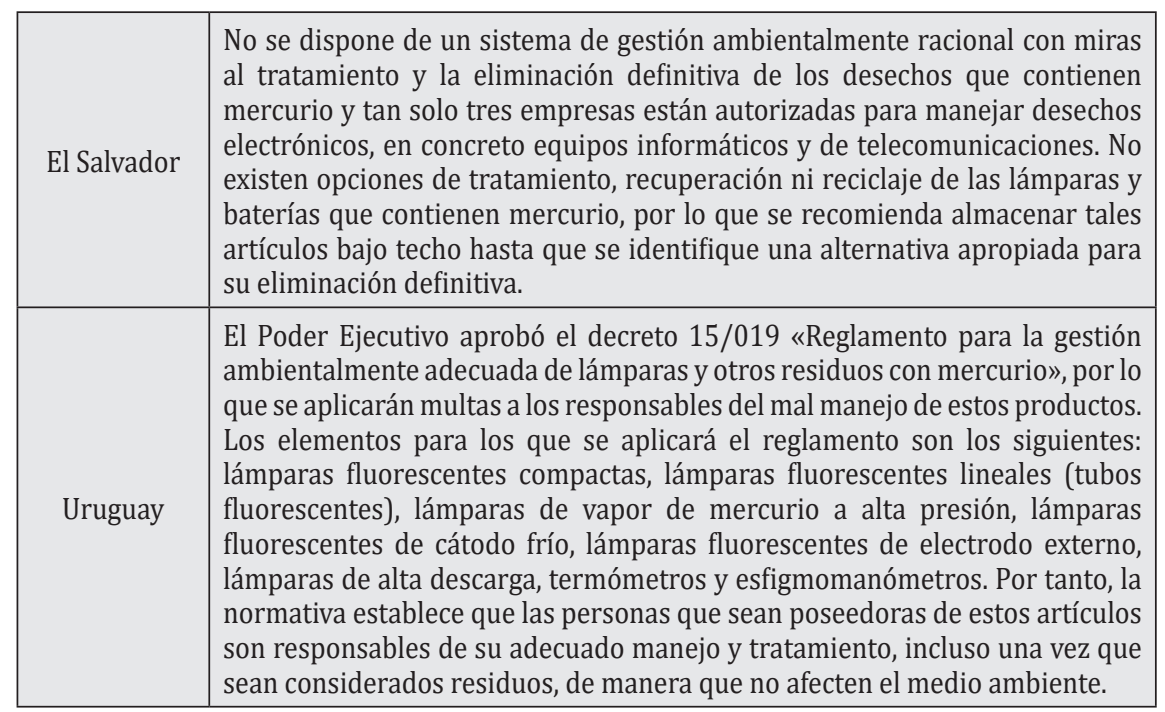

\subsection{América del Norte}

\begin{tabular}{|c|l|}
\hline \multirow{5}{*}{ Canadá } & $\begin{array}{l}\text { Los bienes de consumo que contienen mercurio pueden depositarse en } \\
\text { instalaciones de recogida de desechos peligrosos y centros de distribución } \\
\text { o enviarse a una instalación autorizada de gestión de desechos. Los } \\
\text { subproductos y residuos industriales que contienen mercurio pueden } \\
\text { trasladarse a determinadas instalaciones dentro o fuera de Canadá para su } \\
\text { correcto reciclaje o eliminación. Vertederos diseñados especialmente aceptan } \\
\text { los residuos de mercurio y se encargan de su eliminación (debido a los límites } \\
\text { de lixivialidad, algunas veces el mercurio se solidifica o estabiliza antes de } \\
\text { proceder a su eliminación). Es posible exportar residuos de mercurio desde } \\
\text { Canadá con el objetivo de eliminarlos correctamente o reciclarlos (entre 2010 } \\
\text { y 2015 se exportaron a los Estados Unidos para su tratamiento o eliminación). } \\
\text { De la misma forma, en Canadá es posible importar residuos de mercurio de } \\
\text { otros países para su manejo adecuado y eliminación. }\end{array}$ \\
\hline \multirow{5}{*}{ Estados } \\
Unidos & $\begin{array}{l}\text { Los residuos de mercurio estánsujetos a la Ley deConservación delos Recursos } \\
\text { y de la Recuperación y Restauración Ambiental. El Programa Universal de } \\
\text { Desechos regula la gestión de los residuos peligrosos constituidos por los } \\
\text { dispositivos y lámparas con mercurio que alcanzan el fin de su vida útil. Como } \\
\text { última instancia, estos residuos se recogen de manera selectiva, se recupera } \\
\text { el mercurio (y se almacena), o se tratan y eliminan. Una parte del mercurio se } \\
\text { estabiliza y se exporta a Canadá para su eliminación definitiva en el marco de } \\
\text { las exigencias del Convenio de Basilea. }\end{array}$ \\
\hline
\end{tabular}




\begin{tabular}{|l|l|}
\hline $\begin{array}{l}\text { Los residuos de lámparas fluorescentes se han controlado por el Reglamento } \\
\text { Universal de Residuos (40 CFR part } 273 \text { 2012), la prohibición de productos } \\
\text { con contenido de mercurio y la Ley de Prohibición de Exportación de Mercurio } \\
\text { (Public Law 110-414 2008). Según la Asociación de Iluminación y Reciclaje de } \\
\text { Mercurio, las empresas miembros logran alrededor del 85\% del reciclaje de } \\
\text { las lámparas producidas cada año (Rhee, Choi \& Park 2013). Los requisitos de } \\
\text { tratamiento de residuos con mercurio, de acuerdo a la Restricción de sitios } \\
\text { de disposición (LDR, por sus siglas en inglés), deben ser controlados debajo } \\
\text { del límite de 0,025 mg/L (EPA 2007), tal como se determina en la prueba } \\
\text { Procedimiento de caracterización de toxicidad de lixiviación característico } \\
\text { (TCLP). }\end{array}$ \\
\hline
\end{tabular}

\subsection{Europa}

\begin{tabular}{|c|c|}
\hline $\begin{array}{c}\text { Unión } \\
\text { Europea }\end{array}$ & $\begin{array}{l}\text { En el ámbito de la Unión Europea, actualmente se han retirado numerosos } \\
\text { productos que contienen mercurio; sin embargo, aún está permitido el uso } \\
\text { de ciertas lámparas, cuyo manejo está regulado por la Directiva 2012/19/ } \\
\text { UE sobre residuos de aparatos eléctricos y electrónicos. Dicha directiva } \\
\text { señala que estos residuos deben recogerse por separado y someterse a un } \\
\text { tratamiento específico que reduzca su cantidad, maximice el reciclaje y } \\
\text { garantice la gestión racional de los residuos generados. }\end{array}$ \\
\hline Alemania & $\begin{array}{l}\text { Alemania dispone de tres instalaciones subterráneas capaces de aceptar } \\
\text { residuos de mercurio para su almacenamiento permanente. Los residuos se } \\
\text { sitúan entre } 700 \text { y } 800 \text { metros de profundidad y se aíslan permanentemente } \\
\text { del entorno mediante barreras geológicas y artificiales. Dichas instalaciones } \\
\text { permanecerán aisladas de la biósfera desde su puesta en marcha hasta los } \\
\text { trabajos de mantenimiento posteriores al cierre. Asimismo, cuentan con un } \\
\text { sistema de gestión de calidad (ISO 9001) y con un certificado que acredita su } \\
\text { conformidad con la legislación europea y alemana. }\end{array}$ \\
\hline Austria & $\begin{array}{l}\text { En Austria, cerca de } 40 \text { empresas están involucradas actualmente en el } \\
\text { reciclaje de las lámparas para reciclar vidrio y metales ferrosos, y los residuos } \\
\text { que contienen mercurio son tratados en los sitios de disposición mediante el } \\
\text { uso de un recipiente especial (Rhee et al. 2013). }\end{array}$ \\
\hline España & $\begin{array}{l}\text { La gestión de los residuos de mercurio, incluidas las lámparas fluorescentes, } \\
\text { se realiza de conformidad con la Ley de Residuos y Suelos Contaminados, en } \\
\text { el contexto del reglamento de la Unión Europea. Asimismo, el Real Decreto } \\
\text { sobre aparatos eléctricos y electrónicos, prevé la recogida, el transporte, el } \\
\text { tratamiento y la eliminación. Las lámparas fluorescentes se recogen a través } \\
\text { de unos } 33.300 \text { puntos de recogida, y se tratan en } 5 \text { centros de reciclajes } \\
\text { supervisados por EucoLight, asociación europea de organizaciones de } \\
\text { recogida y reciclaje de equipos eléctricos y electrónicos, lámparas y otros } \\
\text { sistemas de iluminación. España prevé que en estas instalaciones se generen } \\
\text { entre } 900 \text { y } 1.000 \text { toneladas de mercurio elemental y tiene previsto estabilizar } \\
\text { y solidificar el mercurio metálico con vistas a su eliminación ambientalmente } \\
\text { racional en vertederos diseñados especialmente. }\end{array}$ \\
\hline
\end{tabular}




\begin{tabular}{|c|l|}
\hline \multirow{5}{*}{ Suiza } & $\begin{array}{l}\text { Los desechos de mercurio son abordados por la Ley Federal sobre la } \\
\text { Protección del Medio Ambiente. Asimismo, cuenta con una Ordenanza } \\
\text { sobre la Devolución, recuperación y eliminación de los equipos eléctricos y } \\
\text { electrónicos. En el país, los residuos de mercurio doméstico, especialmente las } \\
\text { lámparas fluorescentes, se separan de los otros residuos. Entre las prácticas } \\
\text { habituales se encuentran la devolución de las lámparas fluorescentes al } \\
\text { establecimiento de compra o su depósito en centros de recogida de residuos. } \\
\text { Un porcentaje se trata en Suiza y el resto se exporta a Alemania o Francia para } \\
\text { su posterior tratamiento y eliminación definitiva. }\end{array}$ \\
\hline
\end{tabular}

\subsection{Asia}

\begin{tabular}{|c|l|}
\hline \multirow{6}{*}{ China } & $\begin{array}{l}\text { China lanzó oficialmente un ambicioso programa «Iluminación verde» } \\
\text { (Green Lighting Program 1996), obteniendo un importante ahorro de energía } \\
\text { eléctrica y de emisión de gases de efecto invernadero mediante la sustitución } \\
\text { de lámparas incandescentes por lámparas fluorescentes. El Gobierno anunció } \\
\text { oficialmente en 2011 un plan de acción nacional para la eliminación gradual } \\
\text { de las lámparas incandescentes, sin embargo, el sector de manejo de residuos } \\
\text { sólidos municipales no está preparado para la disposición y tratamiento de } \\
\text { este tipo de lámparas (Hu \& Cheng 2012). }\end{array}$ \\
\hline \multirow{6}{*}{ Japón } & $\begin{array}{l}\text { Los residuos de mercurio se regulan fundamentalmente por la Ley de Gestión } \\
\text { de Desechos y Limpieza Pública. Dichos residuos se tratan principalmente } \\
\text { en instalaciones designadas donde se extrae el mercurio de los residuos. El } \\
\text { mercurio recuperado se utiliza en la producción de productos con mercurio } \\
\text { añadido o se exporta como materia prima. Los residuos de mercurio se } \\
\text { purifican y posteriormente se solidifican con azufre modificado con la } \\
\text { finalidad de eliminarlos en vertederos especialmente diseñados para } \\
\text { ello. Asimismo, el Ministerio del Medio Ambiente promueve la recogida } \\
\text { apropiada de los residuos domésticos que contienen mercurio, así como la } \\
\text { concientización ciudadana en colaboración con los municipios. }\end{array}$ \\
\hline
\end{tabular}

Elaboración propia, adaptado de PNUMA (2017).

\section{Discusión}

La gran mayoría de los países gestionan sus residuos de mercurio como un componente más de los residuos municipales o industriales, y los eliminan en rellenos o botaderos. Algunos países carecen de mecanismos para la recogida selectiva de residuos, mientras que otros, que recogen por separado las lámparas fluorescentes, carecen de una opción de eliminación definitiva en su territorio. En otros países los residuos de mercurio son gestionados como los demás residuos peligrosos. Tan solo un número reducido de países cuenta con tecnología avanzada y los equipos necesarios para tratar los residuos de mercurio, de conformidad con las directrices del Convenio de Basilea. En algunos casos, se dispone de tecnologías y equipos más sencillos para el tratamiento y pretratamiento, como son las trituradoras de lámparas. 
Dentro de las alternativas en el mercado existen varios tipos de máquinas con diferentes alcances, capacidades y posibilidades. Las máquinas más económicas son aquellas que únicamente compactan o trituran las lámparas en un ambiente hermético evitando la emisión de contaminantes al ambiente, y colocan el residuo en un contenedor que posteriormente debe ser llevado a confinamiento o a reciclaje. Además, estas máquinas cuentan con un filtro de carbón activado para atrapar los vapores desprendidos durante la destrucción.

Las máquinas más complejas son las recicladoras que incluyen diferentes tecnologías para la separación de los componentes de las lámparas, haciendo posible la posterior valorización de los componentes (mercurio y vidrio, principalmente). Estas máquinas son costosas y apuntan al tratamiento de una cantidad grande de lámparas para que sea viable la valorización de los componentes y el costo de la máquina.

En 1990, la Agencia de Protección Ambiental (EPA) de los Estados Unidos declaró a las lámparas fluorescentes inservibles como desechos peligrosos que deberían tener un manejo más estricto y riguroso, debido al contenido de mercurio y otros elementos tóxicos, según la Ley de Conservación y Recuperación de Recursos. El elemento mercurio es el más perjudicial de todos los metales, no es esencial para ningún proceso metabólico y es bioacumulable en la mayoría de los seres vivos (UGR citado por Miranda et al. 2015).

La exposición al mercurio contenido dentro de una lámpara se da principalmente por inhalación e ingestión oral, sin embargo, implica mayor riesgo a la salud al ser inhalado, ya que el $80 \%$ de vapor de mercurio inhalado es absorbido por los pulmones, produciendo efectos en el sistema respiratorio como neumonitis química, edema e insuficiencia respiratoria.

A partir de lo señalado, se planteó una propuesta para el manejo de residuos de lámparas en nuestro país, priorizando la valorización de componentes aprovechables de este tipo de residuo, asegurando la disposición final segura de los componentes que no puedan ingresar a una nueva cadena productiva. 


\section{Gráfico 4.}

Propuesta de manejo de las lámparas en el Perú

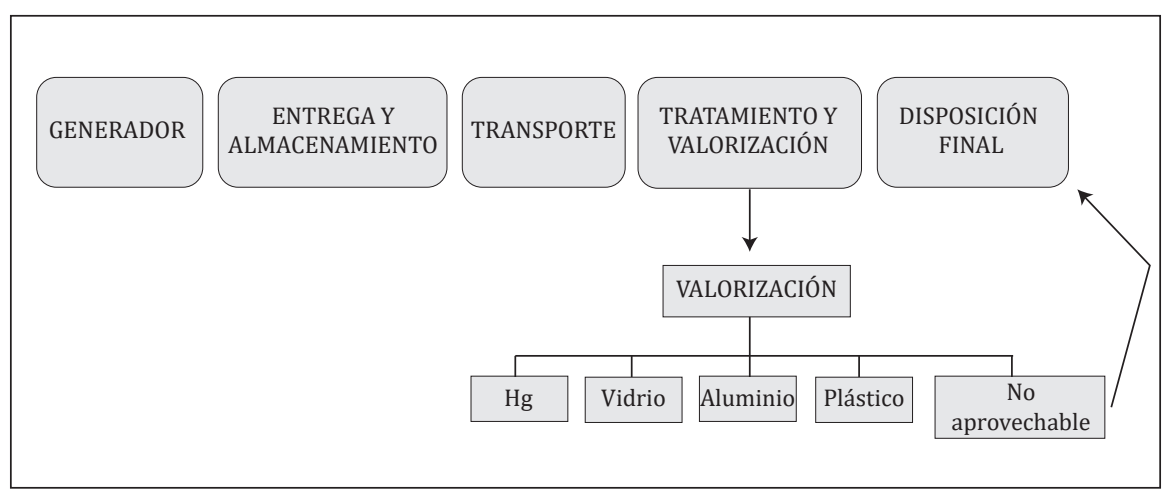

Elaboración propia.

\section{Conclusiones}

Las lámparas fluorescentes, al finalizar su vida útil, constituyen un peligro debido a su contenido de mercurio, su manejo adecuado es un factor importante para reducir los riesgos a la salud humana y al ambiente. En vista del riesgo que representa el mercurio, diversos países del mundo han asumido compromisos mediante la firma del Convenio de Minamata. Sin embargo, en el Perú se viene aún elaborando el Plan Nacional de Aplicación del Convenio de Minamata.

Asimismo, en el Perú la problemática de las lámparas fluorescentes en desuso radica en que son manejadas como un residuo convencional y no como un residuo de aparato eléctrico y electrónico, el cual requiere de un manejo especial (bienes priorizados). Esto se debe a la falta de conocimiento por parte de la población, la inexistencia de campañas de recuperación específicas para luminarias, la insuficiencia de alternativas tecnológicas disponibles para la valorización del residuo, la falta de sanciones por incumplimiento, entre otras. Debido a ello, se plantea una propuesta de manejo de las lámparas que contienen mercurio.

\section{Recomendaciones}

- Siendo el mercurio un elemento tóxico, las autoridades nacionales en materia ambiental deben formular normas que regulen y exijan un 
tratamiento adecuado para los residuos que contengan dicho metal pesado y así evitar los impactos a la salud y al ambiente.

- Se debe contar con normas técnicas para la implementación de plantas de valorización de residuos de aparatos eléctricos y electrónicos, determinando el espacio, áreas específicas para cada proceso, medidas de seguridad, equipos y maquinarias, entre otras cosas; con el fin de que estas especificaciones se deban cumplir por aquellas empresas operadoras especializadas en la valorización de residuos de aparatos eléctricos y electrónicos.

- Las empresas que realicen operaciones de valorización de residuos de aparatos eléctricos y electrónicos deben contar con personal calificado y tecnología adecuada para llevar a cabo las operaciones de valorización, que implica la recuperación de componentes aprovechables que puedan ingresar a una cadena de valor.

- Regular la identificación del mercurio en productos mediante etiquetado y su contenido en los mismos, estableciendo límites permitidos, para facilitar la prevención, reducción y gestión adecuada de los residuos con mercurio.

- Difundir y promover la compra de alternativas al uso de las lámparas convencionales que contengan mercurio, como por ejemplo las lámparas led.

\section{Referencias}

40 CFR part 273 (2012). Part 273 Standards for Universal Waste Management. USA. Disponible en http://www.ecfr.gov/cgi-bin/textidx?tpl=/ecfrbrowse/ Title40/40cfr273_main_02.tpl

Aucott M., McLinden M., \& Winka M. (2003). Release of mercury from broken fluorescent bulbs. J Air Waste Manag Assoc, 53(2):143-151. DOI: 10.1080/10473289.2003.10466132

DIGESA (27 de marzo de 2019). Registro de Empresas Comercializadoras de Residuos Sólidos EC-RS. Obtenido de http://www.digesa.minsa.gob.pe/ Expedientes/Busquedas.asp

DIGESA (27 de marzo de 2019). Registro de Empresas Prestadoras de Servicios de Residuos Sólidos EPS-RS. Obtenido de http://www.digesa.minsa.gob.pe/ Expedientes/Busquedas.asp

EPA (1994). Evaluation of mercury emissions from fluorescent lamp crushing. 
EPA (2007). Title 40 Protection of Environment. Chapter I Environmental Protection Agency. Subchapter I Solid Wastes Part 268.40 - Land Disposal Restrictions. Subpart D - Treatment Standards. USA. Disponible en http://www.gpo.gov/fdsys/pkg/CFR2012-title40-vol28/pdf/CFR-2012title40-vol28-sec268-40.pdf

Gaioli, M., Amoedo, D., \& GonzÁles, D. (2012) Impacto del mercurio sobre la salud humana y el ambiente. Arch Argent Pediatr, 110(3): 259-264. doi. org/10.5546/aap.2012.259

GAONA, X. (2004). El mercurio como contaminante global: Desarrollo de metodologías para su determinación en suelos contaminados y estrategias para la reducción de su liberación al medio ambiente. Tesis de la Universidad Autónoma de Barcelona. Dpto. Química. Unidad Química Analítica.

Green Lighting Program. (1996). China. Disponible en http://projects.wri.org/ sd-pams-database/china/green-lighting-program

Hu, Y., Cheng, H. (2012) Mercury risk from fluorescent lamps in China: Current status and future perspective. Environment International vol. 44 p. 141150. Disponible en http://www.bidi.uam.mx:2088/science/article/pii/ S0160412012000165

Instituto Cuanto (2015). Estudio de Mercado de Iluminación en el Perú. Lima, Perú.

Martineza, N., Ayrala, M., \& Zurbriggen, N. (2012). Lineamiento para la gestión integral de lámparas con contenido de mercurio como residuo peligroso en la República de Argentina. VII Congreso de Medio Ambiente.

MINAM (27 de marzo de 2019). Listado de empresas operadoras de residuos sólidos autorizados por el MINAM. Obtenido de http://www.minam.gob. pe/gestion-de-residuos-solidos/registro-de-empresas-operadoras-deresiduos-solidos-autorizados-por-el-minam/

Miranda, J., Martínez, S. \& Hernández, J. (2015). Análisis del tratamiento y nivel de contaminantes por lámparas fluorescentes en El Salvador. Revista Entorno, (59): 97-110. Universidad Tecnológica de El Salvador. ISSN: 2218-3345.

MVOTMA (2017). Guía para el almacenamiento de residuos con mercurio Pautas para embalaje, etiquetado y almacenamiento de mercurio. Montevideo.

OMS (31 de marzo de 2017). Organización Mundial de la Salud. Obtenido de https://www.who.int/es/news-room/fact-sheets/detail/mercury-andhealth 
Ortega, J., Ferrís, J., López, J., García, J., Cánovas, A., Marco, A., Ortí, A., Ibiza, E., Molina, F., Lorente, D. (2003). Hospitales sostenibles (II). Mercurio: exposición pediátrica. Efectos adversos en la salud humana y medidas preventivas. Revista española de pediatría, 274-291.

PNUMA (2002). Evaluación mundial sobre el mercurio. Ginebra.

PNUMA (2017). Evaluación mundial de los desechos de mercurio. Nairobi.

Poulin, J., \& GiBB, H. (2008). Mercurio: evaluación de la carga de morbilidad ambiental a nivel nacional. Ginebra: Organización Mundial de la Salud.

Public LAW 110-414. (2008). An Act to prohibit the sale, distribution, transfer, and export of elemental mercury, and for other purposes. USA. Disponible en http://www.gpo.gov/fdsys/pkg/PLAW-110publ414/pdf/PLAW110publ414.pdf

Rhee, S., ChoI, H., \& PARK, H. (2013). Characteristics of mercury emission from linear type of spent fluorescent lamp. Waste management. New York. Disponible en http://www.bidi.uam.mx:2088/science/article/pii/ S0956053X13003577

Rice, D. \& BARONE, S. (2000) Critical periods of vulnerability for the developing nervous system: evidence from humans and animal models. Environ Health Perspect. 108 (3):511-533.

SCHER (Scientific Committee on Health and Enviromental Risks) (2010). Opinión on Mercury in Certain Energy-saving Light Bulbs. European Union.

SEMARNAT (2014). Listados de empresas autorizadas. Disponible en http:// tramites.semarnat.gob.mx/index.php/empresas-autorizadas

VARGAS, F. O. (2017). Gestión Ambiental del Manejo de Residuos de Aparatos Eléctricos y Electrónicos (RAEE) provenientes de la comercialización en Tiendas por Departamento. Tesis magistral, Pontificia Universidad Católica del Perú, Lima. 